\title{
Planejamento de médio prazo: panorama de sua aplicação na construção civil
}

\author{
Lookahead planning: overview of its application in \\ construction
}

\section{Vanessa Lira Angelim \\ Thais da Costa Lago Alves \\ Mariana Monteiro Xavier de Lima \\ J osé de Paula Barros Neto}

\section{Resumo

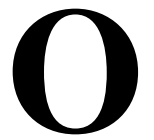

planejamento de médio prazo (PMP) consiste em um nível hierárquico de planejamento do Last Planner System (LPS) e destaca-se como estratégia primordial para garantir a concretização dos planos estabelecidos no planejamento de longo prazo no nível operacional de empreendimentos de construção. Este artigo descreve uma revisão sistemática da literatura (RSL) cujo objetivo foi identificar as principais práticas do PMP, as principais falhas em sua implementação e as lacunas de conhecimento sobre esse tema. Os resultados obtidos apontam a existência de amplo conjunto de práticas no PMP, que podem ser consideradas em futuras pesquisas, bem como incorporadas no processo de planejamento e controle da produção por empresas construtoras. Entretanto, constatou-se generalizada ineficácia em sua implementação. Também foram identificadas lacunas na literatura referentes ao impacto do PMP no desempenho das construções e quanto à aplicação do Building Information Modeling (BIM) para apoiar as funções do PMP. Conclui-se que são necessárias mais pesquisas que contribuam para aumentar a eficácia do PMP nos empreendimentos de construção, dado seu reconhecido impacto na remoção de restrições e, em consequência, na obtenção de fluxo ininterrupto das atividades.

Palavras-chave: Planejamento de médio prazo. Last Planner System (LPS). Planejamento de obras. Controle da produção.

\section{Abstract}

Medium-term planning (MTP) is one of the hierarchical levels of the Last Planner System of production control, and constitutes an essential step to assure that plans defined at the long-term planning level will materialise at the operational level in construction projects. This paper describes a systematic literature review, whose objective is to identify the main practices associated with MTP, the main failures related to its implementation, and existing gaps on this topic. The results point to a plethora of practices associated with MTP, which can be considered in future studies, and also be incorporated in the production planning and control process used by construction companies. However, in general terms, the results of this study show that MTP practices are not being properly implemented. Additionally, the study identified gaps in the literature regarding the impact of MTP on project performance and the use of building information modeling (BIM) to support MTP functions. Hence, this topic merits additional research to improve the efficacy of MTP in construction projects, considering that it represents an essential step to remove project constraints and, consequently, to achieve a continuous flow of activities during the life of a project.

Keywords: Medium-term planning. Last Planner System (LPS). Construction planning. Production control. 


\section{Introdução}

O planejamento de médio prazo (PMP) representa uma etapa fundamental para obter a confiabilidade das previsões e garantir a estabilidade do fluxo de trabalho durante a execução de obras (BALLARD, 1997; HAMZEH; BALLARD; TOMMELEIN, 2008; HAMZEH; BALLARD; TOMMELEIN, 2012; SAMUDIO; ALVES, 2012). Corresponde a um nível hierárquico do processo de planejamento e controle do Last Planner System (LPS), apresentado por Ballard (2000).

O PMP tem função primordial para o sucesso de um empreendimento, visto que contribui para proteger a produção de variações no fluxo de recursos necessários para a realização da obra. Essa analogia é discutida por Ballard e Howell (1998), que relacionam as atividades realizadas no PMP com o uso de um escudo para proteger o fluxo de produção (shielding production). O PMP ineficaz impacta diretamente o desempenho do empreendimento, pois reduz a confiabilidade do planejamento, especialmente em situações com elevado grau de incerteza (BALLARD, 1997).

Apesar do importante papel do PMP para o cumprimento dos prazos das construções, diversos trabalhos, tais como Ballard (1997), Hamzeh, Ballard e Tommelein (2008, 2012), Khanh e Kim (2016) e Hamzeh, Zankoul e Rouhana (2015), afirmam que muitas empresas têm implantado o LPS em diferentes países com sucesso apenas nos níveis de longo e curto prazo. O PMP é recorrentemente negligenciado, sendo implementado de forma incompleta (BALLARD, 1997; HAMZEH; BALLARD; TOMMELEIN, 2008, 2012; KHANH; KIM, 2016; HAMZEH; ZANKOUL; ROUHANA, 2015).

Assim, existem desafios associados à implementação do PMP, sendo importante propor medidas capazes de mitigar tais dificuldades, uma vez que falhas no PMP podem contribuir para reduzir a produtividade e comprometer a confiabilidade do sistema de produção (BORTOLAZZA; FORMOSO, 2006; KEMMER et al., 2007; HOWELL; BALLARD; HALL, 2001; WAMBEKE; LIU; HSIANG, 2012; HAMZEH; LANGERUD, 2011; HAMZEH; ZANKOUL; SAKKA, 2016).

Nesse contexto, diante da importância gerencial do nível de PMP para o desempenho dos empreendimentos e do cenário de sua ineficaz aplicação, este artigo faz uma revisão sistemática da literatura com o objetivo de identificar as principais práticas do PMP, as principais falhas em sua implementação e as lacunas de conhecimento sobre o tema.

\section{Referencial teórico}

\section{Last Planner System}

O LPS é um modelo de planejamento e controle da produção fundamentada nos princípios da filosofia da produção enxuta com vistas a promover a estabilidade do sistema de produção por meio do controle da variabilidade e do aumento da confiabilidade do processo de construção (BALLARD, 2000). Um sistema de produção é estável quando se consegue produzir de acordo com o planejado, sendo a estabilidade fundamental para que os processos não sejam frequentemente interrompidos (LIKER, 2005).

Esse sistema adota níveis hierárquicos no planejamento e controle a fim de gerenciar o que deve ser feito no prazo estabelecido (BALLARD, 2000). Pode ser entendido como um conjunto de quatro níveis de planejamento para transformar o que deve ser feito no que pode ser feito (BALLARD; TOMMELEIN, 2016), descritos a seguir:

(a) planejamento de longo prazo (master scheduling): gerencia os grandes marcos da construção, ou seja, apresenta as atividades contidas no escopo do empreendimento. Possui baixo grau de detalhe devido à falta de informações sobre a duração real das atividades e das entregas de recursos (BALLARD; TOMMELEIN, 2016);

(b) planejamento de fase (phase scheduling): correspondente à interface entre o longo e o médio prazo, ou seja, às atividades que devem ser realizadas e que são decompostas dos principais marcos do plano de longo prazo da obra, detalhando as transições entre as fases (BALLARD; TOMMELEIN, 2016). Objetiva produzir um plano para concluir uma fase de trabalho buscando maximizar a geração de valor e integrando todos os envolvidos da fase para que contribuam para a realização do planejamento (BALLARD; HOWELL, 2003);

(c) planejamento de médio prazo (lookahead planning): horizonte no qual são identificadas e removidas as restrições para viabilizar a execução das atividades conforme definido no planejamento de fase. Nesse nível de planejamento também é detalhado o processamento das atividades e definida a logística de execução (BALLARD; TOMMELEIN, 2016); e 
(d) planejamento de curto prazo (commitment planning): as atividades que estão prontas para ser realizadas são incluídas nos planos de curto prazo, geralmente em ciclos semanais. Objetiva orientar a execução da obra e detalhar as atividades com os responsáveis pelas equipes (BALLARD; TOMMELEIN, 2016). Neste nível deve ocorrer a aprendizagem, que objetiva analisar o trabalho realizado do plano semanal por meio da avaliação de indicadores e da investigação da causa raiz para a não execução das atividades no prazo. Esses dados são utilizados para realizar ações que evitem a recorrência de erros no sistema de produção (BALLARD; TOMMELEIN, 2016).

O indicador percentual de planejamento concluído (PPC) mede o nível de confiabilidade dos planos de trabalho semanal e é calculado por meio do percentual do número de tarefas concluídas no prazo em relação ao número de tarefas planejadas. Pesquisas anteriores encontraram relação entre o PPC e a produtividade de trabalho. Howell, Ballard e Hall (2001) observaram melhorias de produtividade da produção à medida que o empreendimento obteve melhorias no indicador PPC. Em outro estudo, Wambeke, Liu e Hsiang (2012) indicaram que empreendimentos com a aplicação do LPS no processo de planejamento de curto prazo alcançaram desempenho de produtividade 35\% maior do que empreendimentos sem a utilização do LPS.

Por conseguinte, esforços são necessários para melhorar o PPC por meio do planejamento do sistema de produção (BALLARD, 1997). Os resultados de Hamzeh e Langerud (2011) e Hamzeh, Zankoul e Sakka (2016) indicam que o adequado processo de identificação e remoção de restrições contribui para aumentar o PPC.

\section{Funções do planejamento de médio prazo}

Entre as principais ações realizadas no PMP, pode-se citar o planejamento da execução do trabalho na melhor sequência e distribuição; o provisionamento de todos os recursos necessários; a correta e transparente designação das providências e tarefas aos responsáveis diretos pela produção; o monitoramento da entrega de todos os materiais necessários no prazo; o acompanhamento das atividades predecessoras que possuem riscos de atrasar e impactar o prazo da obra; e a promoção do agrupamento de atividades que possuem elevada interdependência (BALLARD, 1997).

As principais funções do PMP classificam-se, de acordo com Coelho (2003), em básicas e secundárias. A primeira função básica do PMP é proteger a produção contra a incerteza por meio de três processos: realizar o detalhamento das atividades do nível dos processos ao nível das operações (BALLARD, 1997; BALLARD; HOWELL, 1998; BALLARD, 2000; HAMZEH, 2009); identificar e remover restrições para tornar as tarefas prontas para a execução (BALLARD, 1997; BALLARD; HOWELL, 1998; BALLARD, 2000; HAMZEH, 2009); e efetuar o desenho das operações através de estudos antes da execução (BALLARD, 1997; HAMZEH, 2009).

Coelho (2003) propôs mais duas funções básicas, integrar os níveis de planejamento e efetuar o controle e aprendizado, este último também proposto por Ballard e Howell (1998). Além das funções básicas, Coelho (2003) elencou funções secundárias: analisar os fluxos físicos; contribuir para a gestão de custos; e planejar e controlar a segurança do trabalho.

\section{Interação dos princípios Lean Production e funções do planejamento de médio prazo}

O LPS baseia-se em princípios básicos da filosofia Lean Construction (LC): estabilidade ao sistema de produção, confiabilidade na execução dos processos produtivos, redução da variabilidade e promoção da melhoria contínua (BALLARD, 2000).

A filosofia da Lean Production representa um novo paradigma relacionado à forma de compreender os processos. Segundo Koskela (2000), no modelo tradicional de gestão da produção tem-se como ênfase o controle das atividades de transformação, enquanto na Lean Production considera-se que a produção é composta de dois tipos de atividades, as que agregam e as que não agregam valor.

As atividades que agregam valor são aquelas que transformam materiais ou informações, considerando as necessidades do cliente, enquanto as atividades que não agregam valor consomem tempo, recursos ou espaço, mas atingem o mesmo objetivo (KOSKELA, 1992).

Koskela (1992) propôs onze princípios da construção enxuta, enumerados a seguir:

(a) reduzir a parcela das atividades que não agregam valor; 
(b) aumentar o valor do produto/serviço através da consideração sistemática dos requisitos dos clientes;

(c) reduzir a variabilidade;

(d) reduzir o tempo de ciclo;

(e) simplificar através da redução do número de passos e partes;

(f) aumentar a flexibilidade de saída;

(g) aumentar a transparência do processo;

(h) focar o controle no processo global;

(i) introduzir melhoria contínua no processo;

(j) balancear melhorias nos fluxos e nas conversões; e

(k) realizar benchmarking.

No Quadro 1, adaptado de Angelim et al. (2018), é apresentada a interação entre os onze princípios da LC estabelecidos por Koskela (1992) e as funções do PMP estabelecidas por Coelho (2003) e fundamentada por outros autores.

Na primeira coluna do Quadro 1 são apresentados os onze princípios da LC; na segunda coluna, as funções do PMP, e no interior do quadro são assinaladas as funções do PMP que se relacionam com cada princípio da LC, o que demonstra o forte apoio das funções do PMP em promover a concretização dos princípios LC em empreendimentos de construções.

Quadro 1 - Interação entre os princípios LC e as funções do PMP

\begin{tabular}{|c|c|c|c|c|c|c|c|c|}
\hline $\begin{array}{l}\text { FUNÇÕES DO PMP } \\
\text { (KRINCÍPIOS LC } \\
\text { (KOSKELA, 1992) }\end{array}$ & 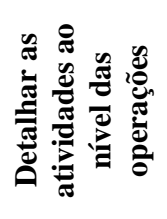 & 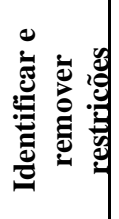 & 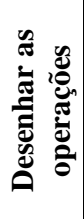 & 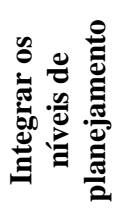 & 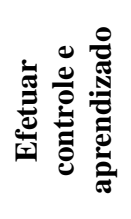 & 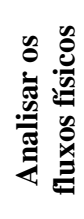 & 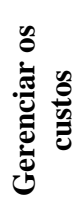 & 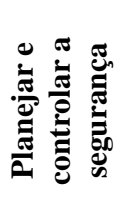 \\
\hline $\begin{array}{l}\text { Reduzir a parcela das } \\
\text { atividades que não agregam } \\
\text { valor }\end{array}$ & $\sqrt{ }$ & & $\sqrt{ }$ & & & $\sqrt{ }$ & & $\sqrt{ }$ \\
\hline $\begin{array}{l}\text { Aumentar o valor do } \\
\text { produto/serviço através da } \\
\text { consideração sistemática dos } \\
\text { requisitos dos clientes }\end{array}$ & $\sqrt{ }$ & & $\sqrt{ }$ & & $\sqrt{ }$ & $\sqrt{ }$ & & $\sqrt{ }$ \\
\hline Reduzir a variabilidade & $\sqrt{ }$ & $\sqrt{ }$ & $\sqrt{ }$ & $\sqrt{ }$ & $\sqrt{ }$ & $\sqrt{ }$ & $\sqrt{ }$ & $\sqrt{ }$ \\
\hline Reduzir o tempo de ciclo & $\sqrt{ }$ & & $\sqrt{ }$ & & $\sqrt{ }$ & $\sqrt{ }$ & & $\sqrt{ }$ \\
\hline $\begin{array}{l}\text { Simplificar através da } \\
\text { redução do número de passos } \\
\text { e partes }\end{array}$ & $\sqrt{ }$ & & $\sqrt{ }$ & & & $\sqrt{ }$ & & $\sqrt{ }$ \\
\hline $\begin{array}{l}\text { Aumentar a flexibilidade de } \\
\text { saída }\end{array}$ & $\sqrt{ }$ & & $\sqrt{ }$ & & & $\sqrt{ }$ & & \\
\hline $\begin{array}{l}\text { Aumentar a transparência do } \\
\text { processo }\end{array}$ & & & $\sqrt{ }$ & $\sqrt{ }$ & $\sqrt{ }$ & $\sqrt{ }$ & & $\sqrt{ }$ \\
\hline $\begin{array}{l}\text { Focar o controle no processo } \\
\text { global }\end{array}$ & & $\sqrt{ }$ & & $\sqrt{ }$ & $\sqrt{ }$ & & $\sqrt{ }$ & \\
\hline $\begin{array}{l}\text { Introduzir melhoria contínua } \\
\text { no processo }\end{array}$ & $\sqrt{ }$ & $\sqrt{ }$ & $\sqrt{ }$ & & $\sqrt{ }$ & $\sqrt{ }$ & & $\sqrt{ }$ \\
\hline $\begin{array}{l}\text { Balancear melhorias nos } \\
\text { fluxos e nas conversões }\end{array}$ & $\sqrt{ }$ & & $\sqrt{ }$ & & & $\sqrt{ }$ & & $\sqrt{ }$ \\
\hline Realizar benchmarking & $\sqrt{ }$ & & $\sqrt{ }$ & & & $\sqrt{ }$ & & \\
\hline
\end{tabular}

Fonte: adaptado de Angelim et al. (2018) 


\section{Método}

A RSL tem como objetivo avaliar estudos primários relacionados a uma questão de pesquisa específica a fim de avaliar criticamente, mapear e consolidar uma síntese dos resultados relevantes desses estudos que resultem em novo conhecimento ou que identifiquem lacunas de conhecimento na área na qual a questão de pesquisa está inserida, tendo como contribuição proporcionar uma visão abrangente e robusta sobre o tema estudado (MORANDI; CAMARGO, 2015).

A condução da revisão sistemática transcorreu com o apoio de uma ferramenta computacional, o State of the Art through Systematic Review (StArt), desenvolvido pelo Laboratório de Pesquisa em Engenharia de Software do Departamento de Computação de São Carlos, SP (FABBRI et al., 2016). Essa ferramenta foi adotada por facilitar a elaboração e o registro do protocolo da RSL, o estabelecimento de critérios para a avaliação dos artigos e o ranqueamento por nível de relevância.

A Figura 1 apresenta o processo metodológico adotado para a RSL e suas etapas. Nesta revisão foi analisada uma série de artigos provenientes das bases de dados disponíveis no Portal de Periódicos da CAPES e do Web of Science, por possuírem ampla abrangência de periódicos internacionais. Também foram analisados artigos publicados pelo International Group for Lean Construction (IGLC), cujos anais reúnem trabalhos específicos da temática abordada.

O protocolo adotado para esta pesquisa se fundamentou na busca de material de estudo nas bases de dados supracitadas segundo o arranjo dos seguintes termos e operadores (busca booleana): "lookahead planning" OR “look-ahead planning” OR “medium-term planning” AND “construction”, como também "lookahead scheduling” OR “look-ahead scheduling” OR “medium-term scheduling” AND construction.

O material de origem das buscas nas bases de dados foi adicionado na ferramenta StArt, na qual os trabalhos foram classificados por ordem de prioridade através de atribuições de escores caso os trabalhos contivessem palavras-chave específicas, inseridas no protocolo do software StArt. Essa classificação por prioridade permitiu auxiliar a avaliação de elegibilidade dos trabalhos, que consiste na leitura do título e do resumo dos estudos, com o objetivo de verificar sua relevância e selecionar os potenciais trabalhos para compor a RSL (DRESCH; LACERDA; ANTUNES JÚNIOR, 2015).

Os critérios de elegibilidade e inclusão na RSL consistiram em trabalhos que contivessem o título e questão de pesquisa específica sobre o PMP, como também trabalhos que abordavam como questão de pesquisa o LPS ou a aplicação dos princípios da LC e que apresentavam informações relevantes em seu texto sobre o uso do PMP em empreendimentos de construção.

Os critérios de exclusão consistiram em se continham informações sem relevância ou artigos sem informações específicas sobre uso do PMP em seus resultados, além de trabalhos duplicados. Portanto, do total de 157 artigos selecionados das bases de dados e inseridos no StArt 61 foram escolhidos. Quinze foram identificados como duplicados e 81 foram rejeitados por não atenderam aos critérios de relevância.

A fase seguinte consistiu na avaliação qualitativa e na síntese dos resultados por meio da leitura completa e analítica dos 61 trabalhos elegíveis, etapa na qual os estudos foram analisados em profundidade (DRESCH; LACERDA; ANTUNES JÚNIOR, 2015). Posteriormente à leitura, os trabalhos foram avaliados quanto a sua relevância, o que é apresentado na Tabela 1.

Nessa etapa mais 11 trabalhos foram rejeitados por não conter informações relevantes sobre o uso do PMP em seus resultados, e 50 foram aceitos para compor a RSL deste trabalho.

Por fim, com base nos estudos selecionados para a RSL, foi realizada a síntese dos dados por meio de leitura acompanhada por síntese dos dados em tópicos, buscando estabelecer relação entre os textos. Com os resultados encontrados, promoveu-se a integração deles, a fim de responder à questão inicial que motivou a RSL, além de gerar novos conhecimentos a partir do conjunto de estudos originais. A estratégia de síntese da RSL consistiu em apontar as semelhanças e diferenças identificadas entre os trabalhos analisados em termos de elementos descritos.

A Figura 2 apresenta a distribuição anual de artigos que possuem resultados sobre o uso do PMP na construção e que foram selecionados para o estudo. Pode-se observar a tendência, ainda que tênue, de aumentar o número de artigos relacionados a esse assunto no período 2011-2016.

Na Tabela 2 são apresentadas as fontes das publicações dos trabalhos selecionados. Observa-se que a maioria dos artigos utilizados na RSL foi publicada nas conferências anuais do IGLC. 
Figura 1 - Metodologia adotada da RSL

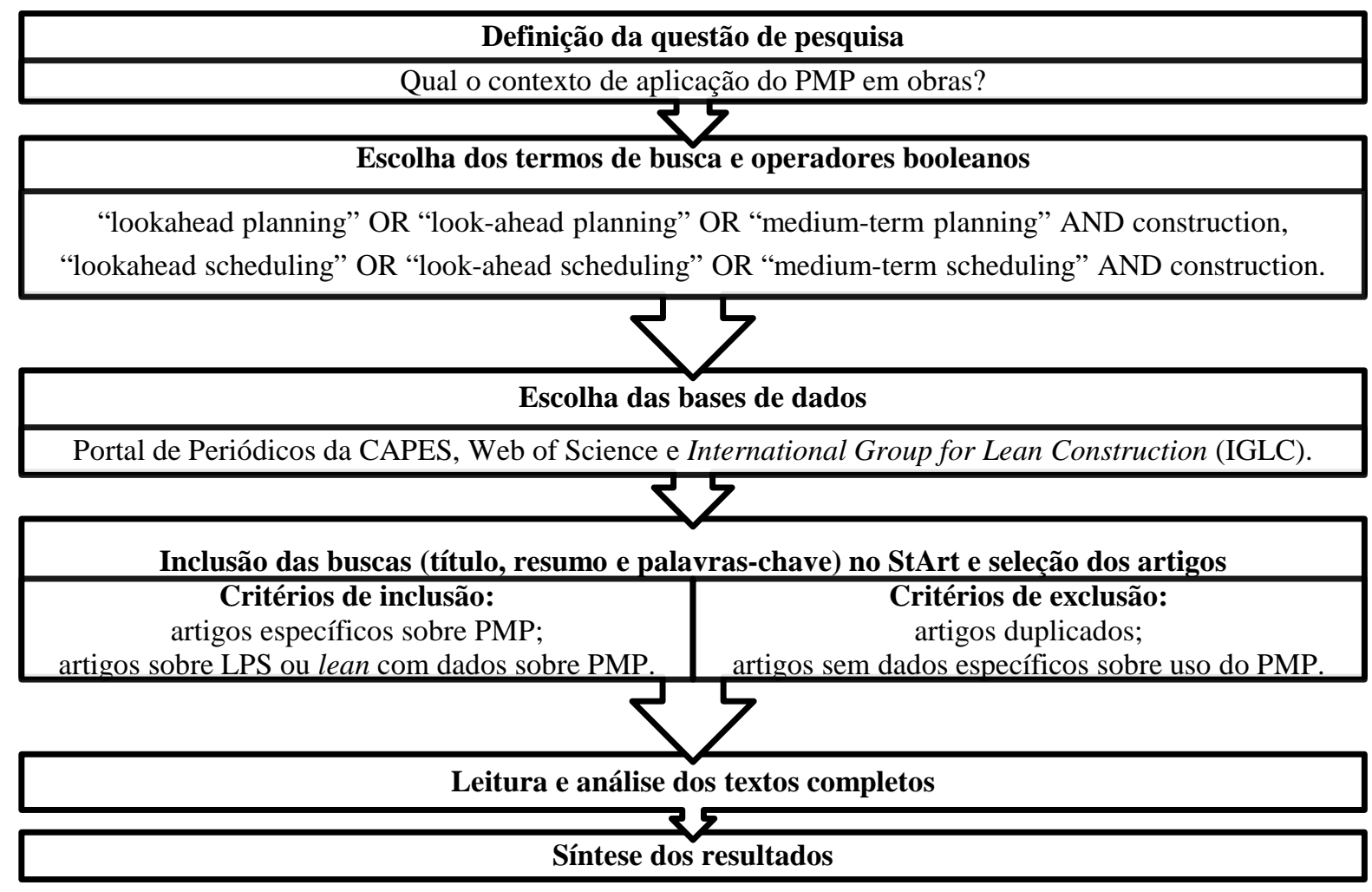

Tabela 1 - Avaliação qualitativa dos trabalhos elegíveis

\begin{tabular}{l|l|c}
\hline \multicolumn{1}{c|}{$\begin{array}{c}\text { Categorias de } \\
\text { relevância }\end{array}$} & \multicolumn{1}{|c}{ Critérios } & $\begin{array}{c}\text { Quantidade de } \\
\text { artigos }\end{array}$ \\
\hline Alta Relevância & Específicos sobre PMP. & 30 \\
\hline Média Relevância & $\begin{array}{l}\text { Tratavam sobre o LPS ou lean com bons resultados } \\
\text { sobre PMP nos resultados. }\end{array}$ & 11 \\
\hline Baixa Relevância & $\begin{array}{l}\text { Tratavam sobre LPS ou lean com baixo nível de } \\
\text { informações sobre PMP nos resultados. }\end{array}$ & 9 \\
\hline Total & \multicolumn{1}{|c|}{50} \\
\hline
\end{tabular}

Figura 2 - Distribuição anual de artigos que abordam planejamento de médio prazo

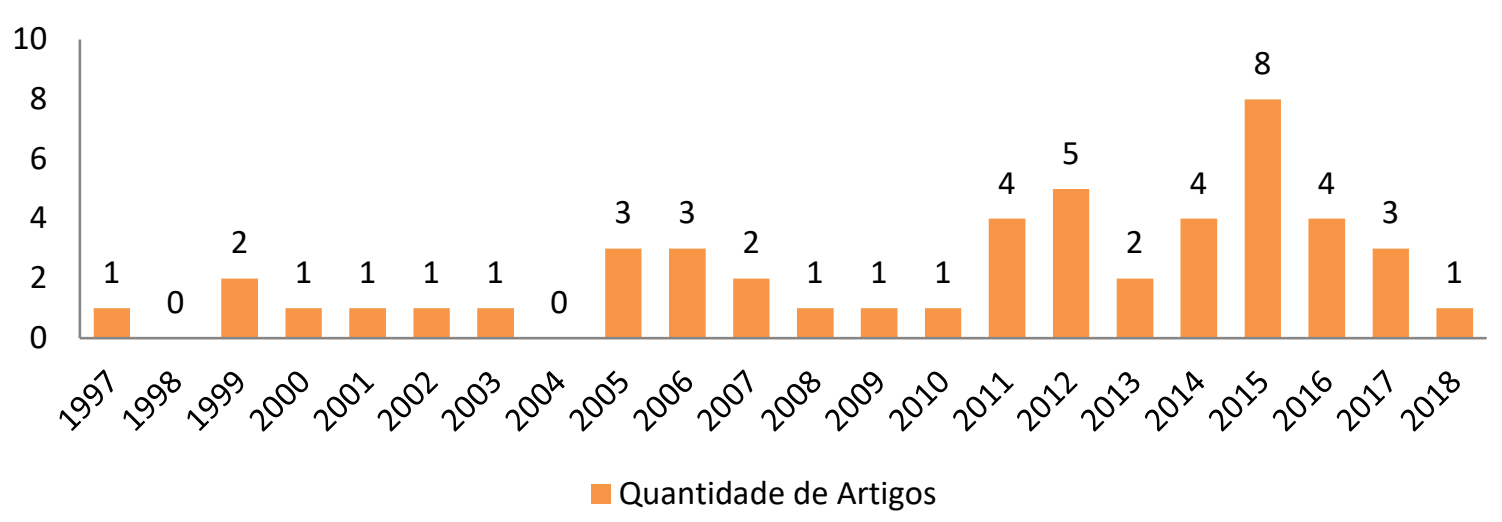


Tabela 2 - Fontes das publicações dos trabalhos da revisão sistemática da literatura

\begin{tabular}{|c|c|c|c|}
\hline $\mathbf{N}^{\mathbf{0}}$ & Fonte da publicação & Total & Qualis ${ }^{1}$ \\
\hline 1 & IGLC & 32 & \\
\hline 2 & Automation in Construction & 6 & A1 \\
\hline 3 & Lean Construction Journal & 3 & \\
\hline 4 & Engineering, Construction and Architectural Management & 2 & B1 \\
\hline 5 & Journal of Computing in Civil Engineering & 1 & \\
\hline 6 & Journal of Construction Engineering and Management & 1 & A1 \\
\hline 7 & AdvancedEngineeringInformatics & 1 & \\
\hline 8 & Construction Management and Economics & 1 & \\
\hline 9 & Procedia Engineering & 1 & \\
\hline 10 & KSCE Journal of Civil Engineering & 1 & $\mathrm{~B} 2$ \\
\hline 11 & Computing in Civil and Building Engineering & 1 & \\
\hline \multicolumn{2}{|c|}{ Total } & 50 & \\
\hline
\end{tabular}

\section{Resultados e discussões}

Na RSL foi considerado o conjunto de artigos apresentados no Quadro 2, classificados por nível de relevância. Foram qualificados 30 artigos como de alta relevância, ou seja, trabalhos que possuem título e questão de pesquisa específica sobre o PMP. Esses trabalhos contribuíram com diversas práticas e lições aprendidas, e serviram para identificar lacunas de conhecimento da pesquisa.

Os artigos classificados como de média relevância, em geral, corresponderam a estudos sobre a adoção do LPS com importantes dados sobre o uso do PMP em seus resultados, os quais, principalmente, contribuíram para compor o conjunto de práticas utilizadas no PMP.

Os trabalhos classificados como de baixa relevância contribuíram para estatísticas sobre a utilização do PMP em diversos países e para compor o conjunto de práticas utilizadas no PMP. Estes corresponderam a estudos sobre o LPS com baixo nível de informações sobre o nível de PMP em seus resultados e artigos que estudaram a integração do LPS com Location-Based Management System (LBMS), técnica de cronograma utilizada no planejamento de longo prazo (SEPPÄNEN; MODRICH; BALLARD, 2010, 2015). Os estudos sobre a integração do LPS e do LBMS buscaram contribuir para a integração entre o longo, fase, médio e curto prazo, como também verificar o impacto no horizonte de PMP por meio da atualização do progresso da construção na LBMS.

\section{Principais falhas observadas durante a execução do PMP}

A RSL mostrou que até em estudos recentes, na prática atual em muitos empreendimentos de construção, há fraca implementação do PMP. O trabalho "Lookahead Planning: The Missing Link in Production Control" enfatiza claramente que o PMP é a ligação negligenciada no controle da produção entre os níveis de planejamento de longo e de curto prazo (BALLARD, 1997). A mesma conclusão é encontrada em trabalhos mais recentes, como em Hamzeh, Ballard e Tommelein (2012), Hamzeh et al. (2015), Hamzeh, Zankoul e Rouhana (2015) e Hamzeh, Zankoul e Sakka (2016).

Foram identificados trabalhos que contribuíram para panorama sobre a utilização do PMP em empreendimentos de construção em todo o mundo. Podem-se citar os trabalhos de Mohan e Iyer (2005) e Salvatierra et al. (2015), que avaliaram a adoção dos princípios Lean em diversas construções. Entre 16 obras distribuídas na Ásia, Américas do Norte e do Sul e Europa, apenas 8 utilizavam o PMP (MOHAN; IYER, 2005). Outro estudo apresentou que de 10 empresas de construção no Chile apenas 2 identificavam e removiam sistematicamente restrições durante a realização do PMP (SALVATIERRA et al., 2015).

Outros trabalhos, como Bortolazza e Formoso (2006), Kemmer et al. (2007), Hamzeh et al. (2012), Wesz, Formoso e Tzortzopoulos (2013), Khanh e Kim (2016), Toledo, Olivares e Gónzalez (2016), realizaram, por meio de survey e de estudos de caso, a análise da aplicação do LPS em empreendimentos com origem em diversos países, como Vietnã, Brasil, Líbano e Chile.

Os resultados dos trabalhos supracitados convergem para uma dificuldade comum na aplicação do LPS. Todas as pesquisas indicam que a maioria dos empreendimentos ainda tem sucesso limitado ao implementar o PMP. 
Diversas falhas nesse nível de planejamento e controle que limitam a eficácia do LPS são apresentadas no Quadro 3.

\section{Lacunas de conhecimento identificadas}

A RSL permitiu identificar lacunas de conhecimento na literatura sobre o tema, que corresponderam ao pequeno número de trabalhos sobre a aplicação de métricas específicas de PMP em empreendimentos de construção e a integração do LPS com o BIM especificamente no que se refere em utilizar o BIM para apoiar o PMP.

Para monitorar o progresso e a melhoria do PMP, Ballard (1997) propôs métricas de medição para verificar seu desempenho, como Tarefas Antecipadas (Tasks Anticipated - TA) e Tarefas Prontas para Execução (Tasks Made Ready-TMR). A TA mede a porcentagem de atividades preparadas para a execução 2 semanas antes do prazo previsto para seu início, garantindo a capacidade de transformar o trabalho que deve ser feito em trabalho que pode ser feito (BALLARD, 1997; HAMZEH; BALLARD; TOMMELEIN, 2008; HAMZEH; LANGERUD, 2011; HAMZEH et al., 2015). A TMR indica a porcentagem de tarefas inseridas no plano semanal de curto prazo que foram preparadas no horizonte de PMP com 2 semanas de antecipação. A TMR é importante para avaliar a qualidade do PMP e medir o desempenho do PMP na identificação e eliminação de restrições para tornar as tarefas prontas para a execução, além de medir a eficácia do PMP em apoiar o plano semanal (BALLARD, 1997; MITROPOULOS, 2005; JANG; KIM, 2007; HAMZEH; BALLARD; TOMMELEIN, 2008; HAMZEH; ZANKOUL; ROUHANA, 2015; HAMZEH; ZANKOUL; SAKKA, 2016).

Quadro 2 - Artigos da RSL

\begin{tabular}{|c|c|c|}
\hline $\begin{array}{l}\text { Categoria de } \\
\text { Relevância }\end{array}$ & $\begin{array}{c}\text { Conjunto de Temas } \\
\text { Abordados }\end{array}$ & Autores \\
\hline $\begin{array}{c}\text { Alta } \\
\text { Relevância }\end{array}$ & $\begin{array}{l}\text { Específicos sobre PMP, } \\
\text { artigos com dados sobre } \\
\text { aplicação do PMP, métricas } \\
\text { de planejamento, restrições, } \\
\text { uso do BIM e gestão de } \\
\text { riscos com o PMP }\end{array}$ & $\begin{array}{l}\text { Ballard (1997), Chua, Jun e Hwee (1999), Jun, Chua e } \\
\text { Hwee (2000), Chua e Shen (2001), Chua, Shen e Hwee } \\
\text { (2003), Mitropoulos (2005), Kim e Jang (2006), } \\
\text { Kemmer et al. (2007), Jang e Kim (2007), Hamzeh, } \\
\text { Ballard e Tommelein (2008), Davis (2009), Alves e } \\
\text { Britt (2011), Hamzeh e Langerud (2011), Dong et al. } \\
\text { (2012), Hamzeh et al. (2012), Song e Eldin (2012), } \\
\text { Hamzeh, Ballard e Tommelein (2012), Samudio e Alves } \\
\text { (2012), Dong et al. (2013), Hamzeh e Aridi (2013), } \\
\text { Wehbe e Hamzeh (2013), Britt et al. (2014), Toledo et } \\
\text { al. (2014), Vahdatikhaki e Hammad (2015), Hamzeh et } \\
\text { al. (2015), Hamzeh, Zankoul e Rouhana (2015), } \\
\text { Emdanat e Azambuja (2016), Hamzeh, Zankoul e Sakka } \\
\text { (2016), Al Hattab, Zankoul e Hamzeh (2017) e Zeng, } \\
\text { König e Teizer (2017) }\end{array}$ \\
\hline $\begin{array}{c}\text { Média } \\
\text { Relevância }\end{array}$ & $\begin{array}{l}\text { Artigos que avaliaram a } \\
\text { adoção do LPS em } \\
\text { empreendimentos, } \\
\text { integração do LPS com } \\
\text { gestão da segurança ou com } \\
\text { o BIM, com bons } \\
\text { resultados em relação ao } \\
\text { PMP }\end{array}$ & $\begin{array}{l}\text { Mendez Junior e Heineck (1999), Saurin et al. (2002), } \\
\text { Bortolazza e Formoso (2006), Sacks, Radosavljevic e } \\
\text { Barak (2010), Antillón et al. (2011), Dave, Boddy e } \\
\text { Koskela (2011), Bhatla e Leite (2012), Dave, Boddy e } \\
\text { Koskela (2013), Dave et al. (2016), Khanh e Kim } \\
\text { (2016), Toledo, Olivares e Gónzalez (2016) }\end{array}$ \\
\hline $\begin{array}{c}\text { Baixa } \\
\text { Relevância }\end{array}$ & $\begin{array}{l}\text { Artigos que avaliaram a } \\
\text { adoção do LPS em } \\
\text { empreendimentos e } \\
\text { integração do LPS com } \\
\text { Location-Based } \\
\text { Management System } \\
\text { (LBMS) com poucos dados } \\
\text { sobre o PMP }\end{array}$ & $\begin{array}{l}\text { Mohan e Iyer (2005), Saurin, Formoso e Cambraia } \\
\text { (2005), Seppänen, Ballard e Pesonen (2010), Wesz, } \\
\text { Formoso e Tzortzopoulos (2013), Scheer et al. (2014), } \\
\text { Seppänen, Modrich e Ballard (2015), Salvatierra et al. } \\
\text { (2015), Dave et al. (2016) e Perez e Ghosh (2018) }\end{array}$ \\
\hline
\end{tabular}




\section{Quadro 3 - Principais falhas observadas durante a execução do PMP}

\begin{tabular}{|c|c|}
\hline Falhas no PMP & Autores \\
\hline $\begin{array}{l}\text { Falta de detalhamento das atividades (usado } \\
\text { apenas para visualizar as atividades do plano- } \\
\text { mestre) }\end{array}$ & $\begin{array}{l}\text { Ballard (1997), Hamzeh, Ballard e Tommelein (2012), } \\
\text { Hamzeh et al. (2015), Hamzeh, Ballard e Tommelein } \\
\text { (2008), Hamzeh et al. (2012), Khanh e Kim (2016) e } \\
\text { Perez e Ghosh (2018) }\end{array}$ \\
\hline Não utilização do planejamento de fase & $\begin{array}{l}\text { Hamzeh, Ballard e Tommelein (2008), Khanh e Kim } \\
\text { (2016) e Perez e Ghosh (2018) }\end{array}$ \\
\hline Falha na identificação e remoção de restrições & $\begin{array}{l}\text { Bortolazza e Formoso (2006), Kemmer et al. (2007), } \\
\text { Hamzeh, Ballard e Tommelein (2008), Wesz, Formoso } \\
\text { e Tzortzopoulos (2013), Salvatierra et al. (2015) e Perez } \\
\text { e Ghosh (2018) }\end{array}$ \\
\hline $\begin{array}{l}\text { Inclusão no plano de curto prazo de atividades } \\
\text { que não estão preparadas para execução }\end{array}$ & $\begin{array}{l}\text { Hamzeh, Ballard e Tommelein (2008), Wesz, Formoso } \\
\text { e Tzortzopoulos (2014) e Hamzeh et al. (2012) }\end{array}$ \\
\hline $\begin{array}{l}\text { Falta de conexão com demais níveis de } \\
\text { planejamento }\end{array}$ & $\begin{array}{l}\text { Hamzeh, Ballard e Tommelein (2008), Hamzeh, Ballard } \\
\text { e Tommelein (2008), Hamzeh e Langerud (2011), } \\
\text { Wesz, Formoso e Tzortzopoulos (2013), Toledo, } \\
\text { Olivares e Gónzalez (2016), Perez e Ghosh (2018) }\end{array}$ \\
\hline $\begin{array}{l}\text { Falta de colaboração entre participantes da } \\
\text { obra }\end{array}$ & $\begin{array}{l}\text { Hamzeh, Ballard e Tommelein (2008) e Britt et al. } \\
\text { (2014) }\end{array}$ \\
\hline $\begin{array}{l}\text { Erro de planejamento (falhas na estimativa de } \\
\text { produtividade dos recursos, agrupamento } \\
\text { ineficaz de tarefas altamente dependentes) }\end{array}$ & $\begin{array}{l}\text { Kemmer et al. (2007), Hamzeh, Ballard e Tommelein } \\
\text { (2008) e Khanh e Kim (2016) }\end{array}$ \\
\hline $\begin{array}{l}\text { Não atribuição correta de responsabilidades } \\
\text { para as ações necessárias no PMP }\end{array}$ & $\begin{array}{l}\text { Hamzeh, Ballard e Tommelein (2008) e Khanh e Kim } \\
\text { (2016) }\end{array}$ \\
\hline $\begin{array}{l}\text { Reuniões de médio prazo improdutivas } \\
\text { (participantes despreparados, solicitações de } \\
\text { informações não prontamente disponíveis) }\end{array}$ & Britt et al. (2014), Toledo, Olivares e Gónzalez (2016) \\
\hline $\begin{array}{l}\text { Não utilização de indicadores para mensurar a } \\
\text { qualidade do PMP }\end{array}$ & $\begin{array}{l}\text { Hamzeh, Ballard; Tommelein (2008), Hamzeh e } \\
\text { Langerud (2011), Hamzeh et al. (2015) e Hamzeh, } \\
\text { Zankoul e Sakka (2016) }\end{array}$ \\
\hline
\end{tabular}

É importante no horizonte de médio prazo gerenciar adequadamente as restrições, designando responsáveis pela remoção e estipulando data-limite para essa ação. Para efetuar esse monitoramento, existe outro indicador, denominado Índice de Remoção das Restrições (IRR), obtido pela razão entre o número de restrições removidas ao final do ciclo do PMP e o número de restrições identificadas para o mesmo período, ou seja, o percentual de restrições identificadas e removidas no prazo (CODINHOTO, 2003).

A revisão da literatura revelou que estudos de casos com foco na mensuração da qualidade do PMP são restritos, pois é difícil encontrar empresas que mensurem indicadores do PMP, como TA e TMR (HAMZEH; BALLARD; TOMMELEIN, 2008; HAMZEH; LANGERUD, 2011; HAMZEH et al., 2015; HAMZEH; ZANKOUL; ROUHANA, 2015). Em contrapartida, o PPC costuma ser a única métrica utilizada que, de forma vaga, representa o progresso geral de planejamento do empreendimento (HAMZEH; BALLARD; TOMMELEIN, 2008; HAMZEH; LANGERUD, 2011).

Diante dessa dificuldade, alguns trabalhos recentes foram desenvolvidos para estudar o PMP e seu impacto em diversas abordagens por meio da simulação computacional (HAMZEH et al., 2015; HAMZEH; LANGERUD, 2011; HAMZEH; ZANKOUL; SAKKA, 2016). Em Hamzeh et al. (2015), a simulação foi utilizada para analisar o efeito do aumento das tarefas antecipadas, mensuradas por TA, na redução da duração da construção. A simulação apontou relação positiva, mas com limite de efeito. Esses autores afirmam que os empreendimentos de construção possuem diferentes níveis de incerteza, o que pode delimitar essa relação. Empreendimentos com atividades repetitivas e com baixa incerteza indicam que a TA aumenta com um bom planejamento. Entretanto, empreendimentos com muitas tarefas interdependentes exigem maior colaboração e esforços no PMP para contribuir no aumento de TA. Hamzeh et al. (2015) salientam que para empreendimentos desse tipo, com maior grau de incerteza, a utilização de BIM e a detecção de interferências nos desenhos dos empreendimentos podem ajudar a aumentar a métrica TA. 
No trabalho de Hamzeh e Langerud (2011), a simulação detectou que o aumento de TA contribui para aumentar o PPC, proporcionando, assim, melhor desempenho do empreendimento e da confiabilidade do fluxo de trabalho. Já no trabalho de Hamzeh, Zankoul e Sakka (2016), a simulação computacional constatou impacto positivo da métrica TMR na execução da tarefa, na confiabilidade do planejamento semanal do trabalho (PPC) e na duração da obra, reafirmando os benefícios do processo de remoção de restrições.

Os autores dos trabalhos de simulação supracitados recomendam que mais empreendimentos comecem a registrar dados para permitir a análise e a replicação experimental. As hipóteses e os resultados desses trabalhos de análise do PMP por simulação requerem validação em empreendimentos reais, com a devida coleta dos indicadores do LPS, integrados com as métricas de progresso de tempo e custo para análise do desempenho da construção. Outra lacuna do conhecimento citada por esses autores é a necessidade de categorizar os tipos de restrições em empreendimentos de construção, para que estes possam ser utilizadas como fonte de dados em futuros empreendimentos.

Embora alguns dos autores supracitados tenham analisado o impacto das métricas TA e TMR no PPC e na duração da construção, há ainda carência de estudos adicionais para testar as hipóteses em função do pequeno número de empreendimentos reais analisados.

Outra lacuna identificada foi a integração do LPS com o BIM especificamente no que se refere ao apoio ao PMP. Dave et al. (2016) e Dave, Boddy e Koskela (2011, 2013) estudaram o VIsiLean, software proposto para fornecer à equipe de construção um sistema de gerenciamento que integre o BIM e o LPS. Sacks, Radosavljevic e Barak (2010) propuseram o KanBim, que consiste em um sistema integrado de andons e fluxos de processos por Kanban, compilados dentro da plataforma BIM, com o objetivo de superar as lacunas entre o LPS e as operações executadas em campo. Toledo, Olivares e Gónzalez (2016) e Bhatla e Leite (2012), por sua vez, abordaram modelos processuais de integração entre o BIM e o LPS a partir da literatura e de estudos de caso.

Bhatla e Leite (2012) realizaram um estudo de caso caracterizado por um empreendimento de construção bemsucedido em termos de implementação do BIM e do LPS. Porém, foram verificados que mais benefícios poderiam ter sido alcançados, principalmente em relação à integração do BIM com o nível de PMP, que não foi realizada de forma sistemática. A utilização do BIM não seguiu um procedimento definido que incorporasse colaboração eficaz entre os participantes da construção. As reuniões de coordenação do BIM eram realizadas separadamente das reuniões de médio prazo. As reuniões de coordenação do BIM não envolviam todos os subcontratados, o que ocasionou falhas de comunicação com esses subcontratados e retrabalho de serviços da obra. Problemas similares foram identificados nos estudos de caso realizados por Toledo, Olivares e Gónzalez (2016). O modelo BIM não foi utilizado durante as reuniões do PMP e não foi compartilhado com todas as partes interessadas do empreendimento.

A pesquisa realizada por Harris e Alves (2016) acerca da utilização do BIM na área de operações revelou que as equipes de obras não aproveitam os benefícios potenciais obtidos com a utilização do BIM no planejamento e na gestão da construção, tais como obter maior nível de visualização e colaboração no empreendimento, melhoria do fluxo de produção, geração de valor e agilidade na resolução de problemas. Constatou-se que os coordenadores de obras, que não trabalham integralmente no local de produção, são os que mais confiam, se beneficiam e utilizam o BIM. Harris (2017) investigou barreiras para a implementação eficaz do BIM na obra e identificou dificuldades relacionadas à baixa quantidade de treinamentos na operação do BIM fornecidos às equipes de produção. Outra dificuldade é a operação do BIM centralizada em uma única pessoa, que geralmente trabalha no escritório da empresa, e não no local da construção. Essa dependência de um profissional externo para gerenciar o BIM resulta em uma divisão entre a obra e a tecnologia virtual, o que pode impedir que os empreendimentos aproveitem os benefícios do BIM em apoiar o planejamento e as operações de produção das construções.

Apenas dois trabalhos buscaram utilizar o BIM no auxílio das funções do PMP: Zeng, König e Teizer (2017) estudaram a gestão de riscos no PMP utilizando o BIM; e Toledo et al. (2014) estudaram restrições com o uso do BIM. Os primeiros autores afirmam que a construção é caracterizada por uma cadeia de suprimentos fragmentada e que envolve diversos participantes. Há envolvidos que trabalham no local da construção, envolvidos externos à produção, participantes da área comercial e de compras que interagem com os participantes anteriores. Diante desse cenário, é necessário existir um gerenciador externo à construção que coordene todas as interações existentes na cadeia de suprimentos durante o PMP com o objetivo de gerenciar efetivamente os riscos inerentes à gestão de suprimentos (ZENG; KÖNIG; TEIZER, 2017).

Portanto, Zeng, König e Teizer (2017) propuseram a utilização do BIM para essa função, em que os riscos identificados no PMP são introduzidos no BIM e representados por um esquema de cores para realizar o 
gerenciamento visual e integrado. Estratégia similar foi identificada no trabalho de Toledo et al. (2014), que utilizaram modelos 4D BIM para visualizar e gerenciar restrições no plano de médio prazo. Estes autores definiram categorias de restrições e atribuíram cores que foram correlacionadas aos objetos no modelo BIM. O objetivo foi exibir visualmente as restrições no PMP, ou seja, as restrições eram representadas na modelagem e sinalizadas aos participantes durante as reuniões de planejamento. Os resultados mostraram que essa ação melhorou o entendimento sobre o gerenciamento e o progresso das restrições do empreendimento por parte dos profissionais envolvidos.

\section{Principais práticas utilizadas no planejamento de médio prazo}

O Quadro 4 apresenta as principais práticas identificadas na literatura, as quais foram classificadas pelas funções básicas do PMP (proteger a produção contra a incerteza, integrar os níveis de planejamento e efetuar controle e aprendizado) e pelas funções secundárias (analisar os fluxos físicos, gerenciar os custos e planejar e controlar a segurança).

\section{Quadro 4 - Principais práticas durante o planejamento de médio prazo coletadas na literatura (Continua...)}

\begin{tabular}{|c|c|c|}
\hline Funções & Práticas Citadas na Literatura & Referências \\
\hline \multirow{5}{*}{$\begin{array}{l}\text { Proteger a } \\
\text { produção contra } \\
\text { a incerteza }\end{array}$} & $\begin{array}{l}\text { Detalhar as tarefas ao nível das operações com melhor } \\
\text { sequência, agrupando trabalho que seja altamente } \\
\text { interdependente }\end{array}$ & $\begin{array}{l}\text { Ballard (1997), Ballard e Howell (1998), } \\
\text { Ballard (2000) e Hamzeh (2009) }\end{array}$ \\
\hline & $\begin{array}{l}\text { Utilizar métodos de automatização para a geração do } \\
\text { PMP e facilitar o processo de proteção da produção } \\
\text { contra a incerteza (facilita o gerenciamento das restrições } \\
\text { e a comunicação entre envolvidos) }\end{array}$ & $\begin{array}{l}\text { Chua, Jun e Hwee (1999), Jun, Chua e } \\
\text { Hwee (2000), Chua e Shen (2001), Chua, } \\
\text { Shen e Hwee (2003), Dong et al. (2012), } \\
\text { Dong (2012), Dong et al. (2013) e Dave et } \\
\text { al. (2016) }\end{array}$ \\
\hline & $\begin{array}{l}\text { Identificar e registrar as restrições por categorias e } \\
\text { responsabilidades }\end{array}$ & $\begin{array}{l}\text { Tommelein e Ballard (1997), Ballard } \\
\text { (2000), Kemmer et al. (2007), Hamzeh } \\
\text { (2009) e Hamzeh et al. (2012) }\end{array}$ \\
\hline & $\begin{array}{l}\text { Avaliar se as tarefas estão prontas para serem agendadas } \\
\text { no curto prazo, excluindo aquelas com pré-requisitos que } \\
\text { faltam, como informações, material, trabalho anterior, } \\
\text { mão de obra e espaço }\end{array}$ & $\begin{array}{l}\text { Tommelein e Ballard (1997), Ballard } \\
\text { (2000), Hamzeh et al. (2012), Kemmer et } \\
\text { al. (2007) e Hamzeh (2009) }\end{array}$ \\
\hline & $\begin{array}{l}\text { Delegar as restrições aos níveis de responsabilidade da } \\
\text { hierarquia organizacional: (1) escritório da empresa, (2) } \\
\text { gerentes ao nível de coordenação de obras e (3) gerente } \\
\text { de linha de frente }\end{array}$ & Kim e Jang (2006) \\
\hline \multirow{6}{*}{$\begin{array}{l}\text { Proteger a } \\
\text { produção contra } \\
\text { a incerteza }\end{array}$} & $\begin{array}{l}\text { Visualizar por cores as restrições no 4D BIM durante } \\
\text { PMP e gerenciar restrições virtualmente em tempo real }\end{array}$ & Toledo et al. (2014) e Alves e Britt (2011) \\
\hline & $\begin{array}{l}\text { Realizar reuniões regulares entre todos os envolvidos, } \\
\text { que devem elaborar seus próprios planos de médio prazo } \\
\text { e questionamentos antes da reunião }\end{array}$ & $\begin{array}{l}\text { Kemmer et al. (2007), Alves e Britt (2011) } \\
\text { e Britt et al. (2014) }\end{array}$ \\
\hline & $\begin{array}{l}\text { Gerenciar riscos da cadeia de suprimentos identificando } \\
\text { os riscos por sinalizadores de cores na modelagem BIM }\end{array}$ & Zeng, König e Teizer (2017) \\
\hline & $\begin{array}{l}\text { Utilizar a técnica de gerenciamento de risco Failure } \\
\text { Mode and Effects Analysis (FMEA), cujas etapas são } \\
\text { identificar os riscos, avaliar e analisar os riscos, } \\
\text { monitorar os riscos e planejar contingências }\end{array}$ & Wehbe e Hamzeh (2013) \\
\hline & $\begin{array}{l}\text { Construir protótipos físicos (unidade do projeto) antes de } \\
\text { iniciar execução }\end{array}$ & $\begin{array}{l}\text { Kemmer et al. (2007) e Hamzeh et al. } \\
\text { (2012) }\end{array}$ \\
\hline & $\begin{array}{l}\text { Construir protótipos virtuais utilizando o BIM (unidade } \\
\text { do projeto) antes de iniciar a execução }\end{array}$ & Hamzeh et al. (2012) \\
\hline
\end{tabular}




\section{Quadro 4 - Principais práticas durante o planejamento de médio prazo coletadas na literatura} (continuação)

\begin{tabular}{|c|c|c|}
\hline \multirow{3}{*}{$\begin{array}{l}\text { Integrar os } \\
\text { níveis de } \\
\text { planejamento }\end{array}$} & $\begin{array}{l}\text { Utilizar a ferramenta Location Based Management } \\
\text { System (LBMS) e obter transparência da integração entre } \\
\text { o longo, fase, médio e curto prazo. Verificar impacto no } \\
\text { PMP através da atualização do progresso no LBMS }\end{array}$ & $\begin{array}{l}\text { Kemmer et al. (2007), Seppänen, Modrich } \\
\text { e Ballard ( 2015), Dave et al. (2016), } \\
\text { Seppänen, Ballard e Pesonen, (2010) }\end{array}$ \\
\hline & $\begin{array}{l}\text { Utilizar indicadores de aderência entre níveis de } \\
\text { planejamento, como o Tasks Made Ready (TMR): \% de } \\
\text { tarefas no plano semanal que foram preparadas no médio } \\
\text { prazo } 2 \text { semanas antes }\end{array}$ & $\begin{array}{l}\text { Ballard (1997), Hamzeh et al. (2012), } \\
\text { Hamzeh (2009), Hamzeh, Zankoul e Sakka } \\
\text { (2016), Hamzeh, Ballard e Tommelein } \\
\text { (2008), Mendez Junior e Heineck (1999), } \\
\text { Hamzeh, Zankoul e Rouhana (2015) e } \\
\text { Hamzeh e Aridi (2013) }\end{array}$ \\
\hline & $\begin{array}{l}\text { Utilizar indicadores de aderência entre níveis de } \\
\text { planejamento: Nível de Compromisso (CL - Commitment } \\
\text { Level): Required Will (atividades requeridas e prontas } \\
\text { para serem executadas no curto prazo) / Should (número } \\
\text { de atividades planejadas no planejamento de fase). Mede } \\
\text { atividades prontas no médio prazo previsto no } \\
\text { planejamento de fase }\end{array}$ & Emdanat e Azambuja (2016) \\
\hline $\begin{array}{l}\text { Efetuar controle } \\
\text { e aprendizado }\end{array}$ & $\begin{array}{l}\text { Utilizar indicador Tasks Anticipated (TA): \% de tarefas } \\
\text { antecipadas } 2 \text { semanas antes da execução ou o Índice de } \\
\text { Remoção das Restrições (IRR): \% de restrições } \\
\text { identificadas e removidas no prazo }\end{array}$ & $\begin{array}{l}\text { Ballard (1997), Hamzeh et al. (2012), } \\
\text { Hamzeh (2009), Hamzeh e Langerud } \\
\text { (2011), Hamzeh et al. (2015), Hamzeh, } \\
\text { Ballard e Tommelein (2008), Hamzeh e } \\
\text { Aridi (2013), Mitropoulos (2005), Jang e } \\
\text { Kim (2007), Codinhoto (2003), Codinhoto } \\
\text { et al. (2003), Coelho (2003), Mitropoulos } \\
\text { (2005) e Jang e Kim (2007) }\end{array}$ \\
\hline \multirow{7}{*}{$\begin{array}{l}\text { Efetuar controle } \\
\text { e aprendizado }\end{array}$} & $\begin{array}{l}\text { Utilizar indicador que verifica se foram identificadas } \\
\text { todas as restrições durante o planejamento: (novas } \\
\text { restrições identificadas durante a execução / total de } \\
\text { restrições identificadas) }\end{array}$ & Mitropoulos (2005) \\
\hline & $\begin{array}{l}\text { Utilizar indicador Percentual de Atividades Planejadas } \\
\text { (PAP): [(Número de Apartamentos Executados) / } \\
\text { (Número de Apartamentos Planejados) -1]. Se PAP =0 } \\
\text { (plano no prazo), se PAP < } 0 \text { (atrasado), se PAP >0 } \\
\text { (adiantado) }\end{array}$ & Mendez Junior e Heineck (1999) \\
\hline & $\begin{array}{l}\text { Analisar as métricas de médio prazo conjuntamente com } \\
\text { os indicadores de desempenho da construção, como a } \\
\text { variação de prazo }\end{array}$ & Mitropoulos (2005) e Hamzeh et al. (2015) \\
\hline & $\begin{array}{l}\text { Subcontratados ter acesso aos planos definidos para os } \\
\text { serviços }\end{array}$ & Kemmer et al. (2007) \\
\hline & $\begin{array}{l}\text { Registrar lições aprendidas - documentar em uma base de } \\
\text { dados as restrições, as perguntas e respostas fornecidas } \\
\text { pelos envolvidos da obra }\end{array}$ & Kemmer et al. (2007) \\
\hline & $\begin{array}{l}\text { Monitorar os planos de médio prazo conjuntamente com } \\
\text { a análise das causas para não conclusão das tarefas } \\
\text { semanais e a evolução do PPC para executar ações } \\
\text { corretivas. }\end{array}$ & $\begin{array}{l}\text { Kemmer et al. (2007), Hamzeh et al. } \\
\text { (2012), Wesz, Formoso e Tzortzopoulos } \\
\text { (2013) }\end{array}$ \\
\hline & $\begin{array}{l}\text { Registrar os eventos de improvisação e correlacionar com } \\
\text { as falhas de curto prazo (falha na conclusão das tarefas } \\
\text { planejadas; falha por incapacidade de planejar as tarefas } \\
\text { durante o PMP e falha por incerteza) }\end{array}$ & Hamzeh et al. (2012) \\
\hline
\end{tabular}




\section{Quadro 4 - Principais práticas durante o planejamento de médio prazo coletadas na literatura} (continuação)

\begin{tabular}{|c|c|c|}
\hline \multirow{4}{*}{$\begin{array}{l}\text { Análise de } \\
\text { fluxos físicos }\end{array}$} & $\begin{array}{l}\text { Analisar leiaute do canteiro - necessidades espaciais para } \\
\text { o armazenamento de materiais e o equipamento } \\
\text { necessário para o transporte }\end{array}$ & Kemmer et al. (2007) \\
\hline & $\begin{array}{l}\text { Direcionar responsável na obra para gerenciar os fluxos } \\
\text { físicos no canteiro de obras (planejamento do } \\
\text { recebimento, estocagem e distribuição pelas equipes de } \\
\text { trabalho) }\end{array}$ & Kemmer et al. (2007) \\
\hline & $\begin{array}{l}\text { Planejar os movimentos dos equipamentos, analisar } \\
\text { riscos relativos a proximidade e condições de simulação } \\
\text { visual do equipamento no canteiro de obra durante a } \\
\text { construção em tempo real, e contribuir para a otimização } \\
\text { de seu uso (exemplos: simulação de máquinas em } \\
\text { construção pesada; simulação da utilização de gruas em } \\
\text { prédios altos) }\end{array}$ & $\begin{array}{l}\text { Vahdatikhaki e Hammad (2015) e Al } \\
\text { Hattab, Zankoul e Hamzeh (2017) }\end{array}$ \\
\hline & $\begin{array}{l}\text { Realizar o rastreamento e simulação em tempo real de } \\
\text { operações de construção pesada para o PMP durante } \\
\text { operações de construção - dados da operação são } \\
\text { constantemente capturados usando sensores de } \\
\text { rastreamento e os dados são então introduzidos em um } \\
\text { modelo de simulação para atualização automática do } \\
\text { modelo }\end{array}$ & Song e Eldin (2012) \\
\hline \multirow{2}{*}{$\begin{array}{l}\text { Gerenciamento } \\
\text { de custos }\end{array}$} & $\begin{array}{l}\text { Desenvolver orçamento da mesma forma em que os } \\
\text { pacotes de trabalho foram planejados - obter informações } \\
\text { de quantidade e custo de forma direta }\end{array}$ & Kemmer et al. (2007) \\
\hline & $\begin{array}{l}\text { Analisar nas reuniões de médio prazo o fluxo de caixa da } \\
\text { obra, renegociando o ritmo dos serviços, datas das } \\
\text { entregas de materiais e contratos com fornecedores }\end{array}$ & Kemmer et al. (2007) \\
\hline \multirow{8}{*}{$\begin{array}{l}\text { Planejamento e } \\
\text { controle de } \\
\text { segurança }\end{array}$} & $\begin{array}{l}\text { Promover gestão da obra comprometida com a segurança } \\
\text { - estabelecer cultura e contratar profissionais para a } \\
\text { segurança conforme exigência por norma }\end{array}$ & Antillón et al. (2011) \\
\hline & $\begin{array}{l}\text { Planejar a segurança do trabalho previamente à execução } \\
\text { da tarefa (em médio prazo) }\end{array}$ & Antillón et al. (2011) \\
\hline & $\begin{array}{l}\text { Envolver subcontratados, que devem ser incluídos nos } \\
\text { treinamentos de segurança em médio prazo }\end{array}$ & Antillón et al. (2011) \\
\hline & $\begin{array}{l}\text { Remover restrições relacionadas à segurança do trabalho } \\
\text { antes que as tarefas façam parte do planejamento de curto } \\
\text { prazo }\end{array}$ & $\begin{array}{l}\text { Antillón et al. (2011), Kemmer et al. } \\
\text { (2007) e Saurin et al. (2002) }\end{array}$ \\
\hline & $\begin{array}{l}\text { Classificar as restrições em cinco categorias de recursos } \\
\text { relacionados à segurança: equipamentos de proteção } \\
\text { coletiva (EPC), equipamentos de proteção individual } \\
\text { (EPI), design de instalações de segurança, treinamento e } \\
\text { espaço }\end{array}$ & Saurin et al. (2002) \\
\hline & $\begin{array}{l}\text { Realizar perguntas sistemáticas nas reuniões de médio } \\
\text { prazo: como os trabalhadores acessam o local de } \\
\text { trabalho? Como os EPC serão instalados? Onde as linhas } \\
\text { de vida estarão ancoradas? }\end{array}$ & Saurin et al. (2002) \\
\hline & $\begin{array}{l}\text { Acompanhar o indicador Trabalhos Executados com } \\
\text { Segurança - \% de pacotes de trabalho executados com } \\
\text { segurança. Identificado no curto prazo (1) nenhuma falha } \\
\text { na concepção dos planos de segurança foi detectada; (2) } \\
\text { não houve falha em sua implementação; e (3) nenhum } \\
\text { acidente ou quase acidentes foram observados }\end{array}$ & $\begin{array}{l}\text { Saurin et al. (2002) e Saurin, Formoso e } \\
\text { Cambraia (2005) }\end{array}$ \\
\hline & $\begin{array}{l}\text { Promover a participação dos trabalhadores na } \\
\text { identificação de riscos de acidente e devidas soluções }\end{array}$ & Saurin et al. (2002) \\
\hline
\end{tabular}

O conjunto de práticas do Quadro 4 pode ser utilizado em futuras pesquisas que busquem solucionar os problemas recorrentes para a ineficaz implementação do PMP, como também podem ser consultado e utilizado por empresas construtoras em seus empreendimentos. 


\section{Conclusões}

O presente trabalho apontou que a implementação do PMP tem sido ineficaz em muitos empreendimentos de construção, mesmo considerando estudos recentes. Diante dessa problemática, foram identificadas falhas recorrentes durante a realização do PMP registradas na literatura.

Uma relevante contribuição deste artigo foi o arcabouço teórico apresentado no Quadro 4, com as principais práticas durante o PMP reveladas pela literatura. O acervo dessas práticas pode ser usado para desenvolver futuras pesquisas, bem como ser incorporado em procedimentos de planejamento e controle da produção por empresas construtoras.

Observa-se que a maior quantidade de publicações refere-se a práticas que tratam da implementação das funções básicas do PMP. As funções secundárias (analisar os fluxos físicos; gerenciar os custos; e planejar e controlar a segurança do trabalho) foram pouco exploradas na literatura, caracterizando-se como uma lacuna de conhecimento.

Este artigo também revela outras lacunas de conhecimento na literatura sobre o tema. Existe a necessidade de estudos que testem hipóteses relacionadas aos impactos do PMP em empreendimentos reais a partir de indicadores de eficácia do PMP, como TA e TRM. Outra lacuna identificada foi a necessidade de utilizar o BIM para apoiar o PMP.

\section{Referências}

AL HATTAB, M.; ZANKOUL, E.; HAMZEH, F. R. Near-real-time optimization of overlapping tower crane operations: a model and case study. Journal of Computing in Civil Engineering, v. 31, n. 4, p. $05017001,2017$.

ALVES, T. da C. L.; BRITT, K. Working to Improve the lookahead plan. In: ANNUAL CONFERENCE OF THE INTERNATIONAL GROUP FOR LEAN CONSTRUCTION, 19., Lima, 2011. Proceedings [...] Lima, 2011.

ANGELIM, V. L. et al. Principais falhas no planejamento de médio prazo de obras: uma revisão sistemática da literatura. In: ENCONTRO NACIONAL DE TECNOLOGIA DO AMBIENTE CONSTRUÍDO, 17., Foz do Iguaçu, 2018. Anais [...] Foz do Iguaçu, 2018.

ANTILLÓN, E. I. et al. A research synthesis on the interface between lean construction and safety management. In: ANNUAL CONFERENCE OF THE INTERNATIONAL GROUP FOR LEAN CONSTRUCTION, 19., Lima, 2011. Proceedings [...] Lima, 2011.

BALLARD, G.; HOWELL, G. A. An update on last planner. In: ANNUAL CONFERENCE OF THE INTERNATIONAL GROUP FOR LEAN CONSTRUCTION, 11., Virginia, 2003. Proceedings [...] Virginia, 2003.

BALLARD, G.; HOWELL, G. A. Shielding production: essential step in production control. Journal of Construction Engineering and Management, v. 124, n. 1, p. 11-17, 1998.

BALLARD, G.; TOMMELEIN, I. Current process benchmark for the Last Planner System. Lean Construction Journal, Berkeley, p. 57-89, 2016.

BALLARD, H. G. Lookahead planning: the missing link in production control. In: ANNUAL CONFERENCE OF THE INTERNATIONAL GROUP FOR LEAN CONSTRUCTION, 5., Gold Coast, 1997. Proceedings [...] Gold Coast, 1997.

BALLARD, H. G. The last planner system of production control. Birmingham, 2000. 192 f. Ph.D., University of Birmingham, Birmingham, 2000.

BHATLA, A.; LEITE, F. Integration framework of BIM with the last planner system. In: ANNUAL CONFERENCE OF THE INTERNATIONAL GROUP FOR LEAN CONSTRUCTION, 20., San Diego, 2012. Proceedings [...] San Diego, 2012.

BORTOLAZZA, R. C.; FORMOSO, C. T. A quantitative analysis of data collected from the last planner system in Brazil. In: ANNUAL CONFERENCE OF THE INTERNATIONAL GROUP FOR LEAN CONSTRUCTION, 14., Santiago, 2006. Proceedings [...] Santiago, 2006. 
BRITT, K. et al. Lessons learned from the make ready process in a hospital project. In: ANNUAL CONFERENCE OF THE INTERNATIONAL GROUP FOR LEAN CONSTRUCTION, 22., Oslo, 2014. Proceedings [...] Oslo, 2014.

CHUA, D. K. H.; JUN, S. Li; HWEE, B. S. Integrated production scheduler for construction look-ahead planning. In: ANNUAL CONFERENCE OF THE INTERNATIONAL GROUP FOR LEAN CONSTRUCTION, 7., Berkeley, 1999. Proceedings [...] Berkeley, 1999.

CHUA, D. K. H.; SHEN, L. J.; HWEE, B. S. Constraint-Based Planning with Integrated Production Scheduler over Internet. Journal of Construction Engineering and Management, v. 129, n. 3, p. 293-301, 2003.

CHUA, D. K. H.; SHEN, L. J. Constraint modeling and buffer management with integrated production scheduler. In: ANNUAL CONFERENCE OF THE INTERNATIONAL GROUP FOR LEAN CONSTRUCTION, 9., Singapore, 2001. Proceedings [...] Singapore, 2001.

CODINHOTO, R. Diretrizes para o planejamento e controle integrado dos processos de projeto e produção na construção civil. Porto Alegre, 2003. 178 f. Dissertação (Mestrado em Engenharia) Programa de Pós-Graduação em Engenharia Civil, Universidade Federal do Rio Grande do Sul, Porto Alegre, 2003.

CODINHOTO, R. et al. Análise de restrições: definição e indicador de desempenho. In: SIMPÓSIO BRASILEIRO DE GESTÃO E ECONOMIA DA CONSTRUÇÃO, 3., São Carlos, 2003. Anais [...] São Carlos, 2003.

COELHO, H. O. Diretrizes e requisitos para o planejamento e controle da produção em nível de médio prazo na construção civil. Porto Alegre, 2003. 135 f. Dissertação (Mestrado em Engenharia) - Programa de Pós-Graduação em Engenharia Civil, Universidade Federal do Rio Grande do Sul, Porto Alegre, 2003.

DAVE, B. et al. Opportunities for enhanced lean construction management using Internet of Things standards. Automation in Construction, v. 61, p. 86-97, 2016.

DAVE, B.; BODDY, S.; KOSKELA, L. Visilean: designing a production management system with lean and BIM. In: ANNUAL CONFERENCE OF THE INTERNATIONAL GROUP FOR LEAN CONSTRUCTION, 19., Lima, 2011. Proceedings [...] Lima, 2011.

DAVE, B.; BODDY, S.; KOSKELA, L. Challenges and opportunities in implementing lean and BIM on an infrastructure project. In: ANNUAL CONFERENCE OF THE INTERNATIONAL GROUP FOR LEAN CONSTRUCTION, 21., Fortaleza, 2013. Anais [...] Fortaleza, 2013.

DAVE, B.; SEPPÄNEN, O.; MODRICH, R.-U. Modeling information flows between last planner and location based management system. In: ANNUAL CONFERENCE OF THE INTERNATIONAL GROUP FOR LEAN CONSTRUCTION, 24., Boston, 2016. Proceedings [...] Boston, 2016.

DAVIS, S. R. An algorithm for creating master schedules that minimises schedule reorganisation resulting from adverse risk events. In: ANNUAL CONFERENCE OF THE INTERNATIONAL GROUP FOR LEAN CONSTRUCTION, 17., Taipei, 2009. Proceedings [...] Taipei, 2009.

DONG, N. et al. A genetic algorithm-based method for look-ahead scheduling in the finishing phase of construction projects. Advanced Engineering Informatics, v. 26, n. 4, p. 737-748, 2012.

DONG, N. Automated look-ahead schedule generation and optimization for the finishing phase of complex construction projects. Stanford, 2012. Ph.D. CIFE Technical Report, Stanford University, 2012.

DONG, N. et al. A method to automate Look-Ahead Schedule (LAS) generation for the finishing phase of construction projects. Automation in Construction, v. 35, p. 157-173, 2013.

DRESCH, A.; LACERDA, D. P.; ANTUNES JÚNIOR, J. A. V. Design science research: método de pesquisa para avanço da ciência e tecnologia. Porto Alegre: Bookman, 2015.

EMDANAT, S.; AZAMBUJA, M. Aligning near and Long Term Planning for LPS implementations: a review of existing and new metrics. Lean Construction Journal, v. 101, p. 90-101, 2016.

FABBRI, S. et al. Improvements in the StArt tool to better support the systematic review process. In: INTERNATIONAL CONFERENCE ON EVALUATION AND ASSESSMENT IN SOFTWARE ENGINEERING, 20., New York, 2016. Proceedings [...] New York: ACM Press, 2016. 
HAMZEH, F. R. et al. Is improvisation compatible with look ahead planning? An exploratory study. In: ANNUAL CONFERENCE OF THE INTERNATIONAL GROUP FOR LEAN CONSTRUCTION 2011, 20., San Diego, 2012. Proceedings [...] San Diego, 2012.

HAMZEH, F. R. et al. Understanding the role of "tasks anticipated” in lookahead planning through simulation. Automation in Construction, v. 49, p. 18-26, 2015.

HAMZEH, F. R. Improving construction workflow: the role of production planning and control. Berkeley, 2009. 271 f. Ph.D. in Engineering - Civil and Environmental Engineering, University of California, Berkeley, 2009.

HAMZEH, F. R.; ARIDI, O. Z. Modeling the last planner system metrics: a case study of an aec company Week. In: ANNUAL CONFERENCE OF THE INTERNATIONAL GROUP FOR LEAN CONSTRUCTION, 21., Fortaleza, 2013. Anais [...] Fortaleza, 2013.

HAMZEH, F. R.; BALLARD, G.; TOMMELEIN, I. D. Improving construction work flow: the connective role of lookahead planning. In: ANNUAL CONFERENCE OF THE INTERNATIONAL GROUP FOR LEAN CONSTRUCTION, 16., Manchester, 2008. Proceedings [...] Manchester, 2008.

HAMZEH, F. R.; BALLARD, G.; TOMMELEIN, I. D. Rethinking lookahead planning to optimize construction workflow. Lean Construction Journal, p. 15-34, 2012.

HAMZEH, F. R.; LANGERUD, B. Using simulation to study the impact of improving lookahead planning on the reliability of production planning. In: WINTER SIMULATION CONFERENCE, Grand Arizona Resor Phoenix, 2011. Proceedings [...] Grand Arizona Resor Phoenix, 2011.

HAMZEH, F. R.; ZANKOUL, E.; ROUHANA, C. How can 'tasks made ready' during lookahead planning impact reliable workflow and project duration? Construction Management and Economics, v. 33, n. 4, p. 243-258, 2015.

HAMZEH, F. R.; ZANKOUL, E.; SAKKA, F. Removing constraints to make tasks ready in weekly work planning. Procedia Engineering, v. 164, p. 68-74, jun. 2016.

HARRIS, B. N. Building Information Modeling and construction operations. San Diego, 2017. 119 f. Master of Science in Civil Engineering, San Diego State University, San Diego, 2017.

HARRIS, B. N.; ALVES, T. da C. L. Building Information Modeling: a report from the field. In: ANNUAL CONFERENCE OF THE INTERNATIONAL GROUP FOR LEAN CONSTRUCTION, 24., Boston, 2016. Proceedings [...] Boston, 2016.

HOWELL, G.; BALLARD, G.; HALL, J. Capacity utilization and wait time: a primer for construction. In: ANNUAL CONFERENCE OF THE INTERNATIONAL GROUP FOR LEAN CONSTRUCTION, 9., Singapore, 2001. Proceedings [...] Singapore, 2001.

ISSA, U. H. Implementation of lean construction techniques for minimizing the risks effect on project construction time. Alexandria Engineering Journal, v. 52, n. 4, p. 697-704, 2013.

JANG, J. W.; KIM, Y. W. Use of percent of constraint removal to measure the make ready process. In: ANNUAL CONFERENCE OF THE INTERNATIONAL GROUP FOR LEAN CONSTRUCTION, 15. East Lansing, 2007. Proceedings [...] East Lansing, 2007.

JUN, S. L.; CHUA, D. H. K.; HWEE, B. S. Distributed scheduling with integrated production scheduler. In: ANNUAL CONFERENCE OF THE INTERNATIONAL GROUP FOR LEAN CONSTRUCTION, 8., Brigthon, 2000. Proceedings [...] Brigthon, 2000.

KEMMER, S. L. et al. Medium-term planning: contributions based on field application. In: ANNUAL CONFERENCE OF THE INTERNATIONAL GROUP FOR LEAN CONSTRUCTION, 13., Sydney, 2007. Proceedings [...] Sydney, 2007.

KHANH, H. D.; KIM, S. Y. A survey on production planning system in construction projects based on Last Planner System. KSCE Journal of Civil Engineering, v. 20, n. 1, p. 1-11, 2016.

KIM, Y. W.; JANG, J. W. Applying organizational hierarchical constraint analysis to production planning. In: ANNUAL CONFERENCE OF THE INTERNATIONAL GROUP FOR LEAN CONSTRUCTION, 14., Santiago, 2006. Proceedings [...] Santiago, 2006. 
KOSKELA, L. An exploration towards a production theory and its application to construction. Espoo, 2000. 298 f. Thesis (Engineering Doctoral Thesis) - Helsinki University of Technology, Espoo, Finland, 2000.

KOSKELA, L. Application of the new production philosophy to construction. Center for Integrated Facility Engineering, v. 72, p. 1-81, 1992.

LIKER J. K. O modelo Toyota: 14 princípios de gestão do maior fabricante do mundo. Trad. Ribeiro L. B. Porto Alegre: Bookman, 2005.

MENDEZ JUNIOR, R.; HEINECK, L. F. M. Towards production control on multi-story building construction sites. In: ANNUAL CONFERENCE OF THE INTERNATIONAL GROUP FOR LEAN CONSTRUCTION, 7., Berkeley, 1999. Proceedings [...] Berkeley, 1999.

MITROPOULOS, P. T. "Planned work ready": a proactive metric for project control. In: 1ANNUAL CONFERENCE OF THE INTERNATIONAL GROUP FOR LEAN CONSTRUCTION, 13., Sydney, 2005. Proceedings [...] Sydney, 2005.

MOHAN, S. B.; IYER, S. Effectiveness of lean principles in construction. In: ANNUAL CONFERENCE OF THE INTERNATIONAL GROUP FOR LEAN CONSTRUCTION, 13., Sydney, 2005. Proceedings [...] Sydney, 2005.

MORANDI, M. I. W. M.; CAMARGO, L. F. R. Revisão sistemática da literatura. In: DRESCH, A.; LACERDA, D. P.; ANTUNES JUNIOR, J. A. V. Design sciencie research: método e pesquisa para avanço da ciência e da tecnologia. Porto Alegre: Bookman, 2015.

PEREZ, A. M.; GHOSH, S. Barriers faced by new-adopter of Last Planner System®: a case study. Engineering, Construction and Architectural Management, v. 25, n. 9, p. 1110-1126, 2018.

SACKS, R.; RADOSAVLJEVIC, M.; BARAK, R. Requirements for building information modeling based lean production management systems for construction. Automation in Construction, v. 19, n. 5, p. 641655, 2010.

SALVATIERRA, J. L. et al. Lean diagnosis for chilean construction industry: towards more sustainable Lean practices and tools. In: ANNUAL CONFERENCE OF THE INTERNATIONAL GROUP FOR LEAN CONSTRUCTION, 23., Perth, 2015. Proceedings [...] Perth, 2015.

SAMUDIO, M.; ALVES, T. da C. L. Look-ahead planning: reducing variation to work flow on projects. In: ANNUAL CONFERENCE OF THE INTERNATIONAL GROUP FOR LEAN CONSTRUCTION, 20., San Diego, 2012. Proceedings [...] San Diego, 2012.

SAURIN, T. A. et al. Safety and production: an integrated planning and control model. In: ANNUAL CONFERENCE OF THE INTERNATIONAL GROUP FOR LEAN CONSTRUCTION, 10., Gramado, 2002. Anais [...] Gramado, 2002.

SAURIN, T. A.; FORMOSO, C. T.; CAMBRAIA, F. B. Analysis of a safety planning and control model from the human error perspective. Engineering, Construction and Architectural Management, v. 12, n. 3, p. 283-298, 2005.

SCHEER, S. et al. On-site BIM model use to integrate 4D/5D activities and construction works: a case study on a Brazilian low income housing enterprise. In: COMPUTING IN CIVIL AND BUILDING ENGINEERING, Reston, 2014. Proceedings [...] Reston, 2014.

SEPPÄNEN, O.; BALLARD, G.; PESONEN, S. The combination of last planner system and location-based management system. Lean Construction Journal, v. 6, n. 1, p. 43-54, 2010.

SEPPÄNEN, O.; MODRICH, R.-U.; BALLARD, G. Integration of last planner system and location-based management system. In: ANNUAL CONFERENCE OF THE INTERNATIONAL GROUP FOR LEAN CONSTRUCTION, 23., Perth, 2015. Proceedings [...] Perth, 2015.

SONG, L.; ELDIN, N. N. Adaptive real-time tracking and simulation of heavy construction operations for look-ahead scheduling. Automation in Construction, v. 27, p. 32-39, 2012.

TOLEDO, M. et al. Using 4D models for tracking project progress and visualizing the owner's constraints in fast-track retail renovation projects. In: ANNUAL CONFERENCE OF THE INTERNATIONAL GROUP FOR LEAN CONSTRUCTION, 22., Oslo, 2014. Proceedings [...] Oslo, 2014. 
TOLEDO, M.; OLIVARES, K.; GÓNZALEZ, V. Exploration of a lean - Bim planning framework: a last planner system and BIM - based case study. In: ANNUAL CONFERENCE OF THE INTERNATIONAL GROUP FOR LEAN CONSTRUCTION, 24., Boston, 2016. Proceedings [...] Boston, 2016.

TOMMELEIN, I. D.; BALLARD, H. G. Look-ahead-planning-screening-and-pulling. In: ANNUAL CONFERENCE OF THE INTERNATIONAL GROUP FOR LEAN CONSTRUCTION, 2., São Paulo, 1997. Anais [...] São Paulo, 1997.

VAHDATIKHAKI, F.; HAMMAD, A. Risk-based look-ahead workspace generation for earthwork equipment using near real-time simulation. Automation in Construction, v. 58, p. 207-220, 2015.

WAMBEKE, B. W.; LIU, M.; HSIANG, S. M. Using last planner and a risk assessment matrix to reduce variation in mechanical related construction tasks. Journal of Construction Engineering and Management, v. 138, n. 4, p. 491-498, 2012.

WEHBE, F. A.; HAMZEH, F. R. Failure mode and effect analysis as a tool for risk management in construction planning. In: ANNUAL CONFERENCE OF THE INTERNATIONAL GROUP FOR LEAN CONSTRUCTION, 21., Fortaleza, 2013. Anais [...] Fortaleza, 2013.

WESZ, J.; FORMOSO, C. T.; TZORTZOPOULOS, P. Design process planning and control: last planner system adaptation. In: ANNUAL CONFERENCE OF THE INTERNATIONAL GROUP FOR LEAN CONSTRUCTION, 21., Fortaleza, 2013. Anais [...] Fortaleza, 2013.

ZENG, N.; KÖNIG, M.; TEIZER, J.. Off-site guarding: look-ahead supply scheduling for risk indication with BIM. In: ANNUAL CONFERENCE OF THE INTERNATIONAL GROUP FOR LEAN CONSTRUCTION, 25., Heraklion, 2017. Proceedings [...] Heraklion, 2017.

Vanessa Lira Angelim

Instituto Federal de Educação, Ciência e Tecnologia do Ceará | Av. Dr. Geraldo Barbosa Marques, 567 | Crateús - CE - Brasil | CEP 63700000 | Tel.: (88) 3692-3864 | E-mail: angelim.vanessa@gmail.com

\section{Thais da Costa Lago Alves}

Construction, and Environmental Engineering Department | San Diego State University | 5500 Campanile Dr. | San Diego - CA - Estados Unidos | Tel.: +1 619-594-8289 | E-mail: talves@sdsu.edu

\section{Mariana Monteiro Xavier de Lima}

Departamento de Arquitetura e Urbanismo e Design | Universidade Federal do Ceará | Av. da Universidade, 2890 | Fortaleza - CE - Brasil | CEP 60020-181 | Tel.: (85) 3366-7955 | E-mail: mariana@daud.ufc.br

\section{J osé de Paulo Barros Neto}

Departamento de Integração Acadêmica e Tecnológica | Universidade Federal do Ceará | Bloco 710, Campus do Pici | Fortaleza - CE Brasil | CEP 60455-900 | Tel.: (85) 3366-9607 | E-mail: barrosneto@gercon.ufc.br

\section{Ambiente Construído}

Revista da Associação Nacional de Tecnologia do Ambiente Construído Av. Osvaldo Aranha, 99 - 3o andar, Centro

Porto Alegre - RS - Brasil CEP $90035-190$

Telefone: +55 (51) 3308-4084

Fax: +55 (51) 3308-4054

www. seer. ufrgs. br/ ambienteconstruido

E-mail: ambienteconstruido@ufrgs.br 